\title{
Stroke syndromes
} associated with DWI-negative MRI include ataxic hemiparesis and isolated internuclear ophthalmoplegia

Jennifer Watts, MBBS

Brad Wood, MBBS, FRANZCR

Andrew Kelly, MBBS, FRACP

Anthony Alvaro, MBBS, FRACP

\section{Summary}

We present a case series of clinically definite acute stroke with negative diffusion-weighted imaging (DWI). This study retrospectively examined a large population of stroke patients with the aim of identifying which stroke syndromes were more likely to be negative on MRI. Patient records and images were reviewed in order to confirm clinically definite stroke and DWI negativity. A total of 701 patients had MRI during the study period. A total of 16 patients with DWI-negative MRI and clinically definite stroke as diagnosed by experienced stroke consultants were identified. A total of 15 of the 16 cases were classified as either posterior circulation or lacunar strokes, and the most common syndromes were ataxic hemiparesis and isolated internuclear ophthalmoplegia.

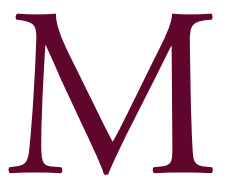

$\mathrm{RI}$ is increasingly used as the investigation of choice for diagnosis of acute ischemic stroke. Diffusion-weighted imaging (DWI) is more sensitive than

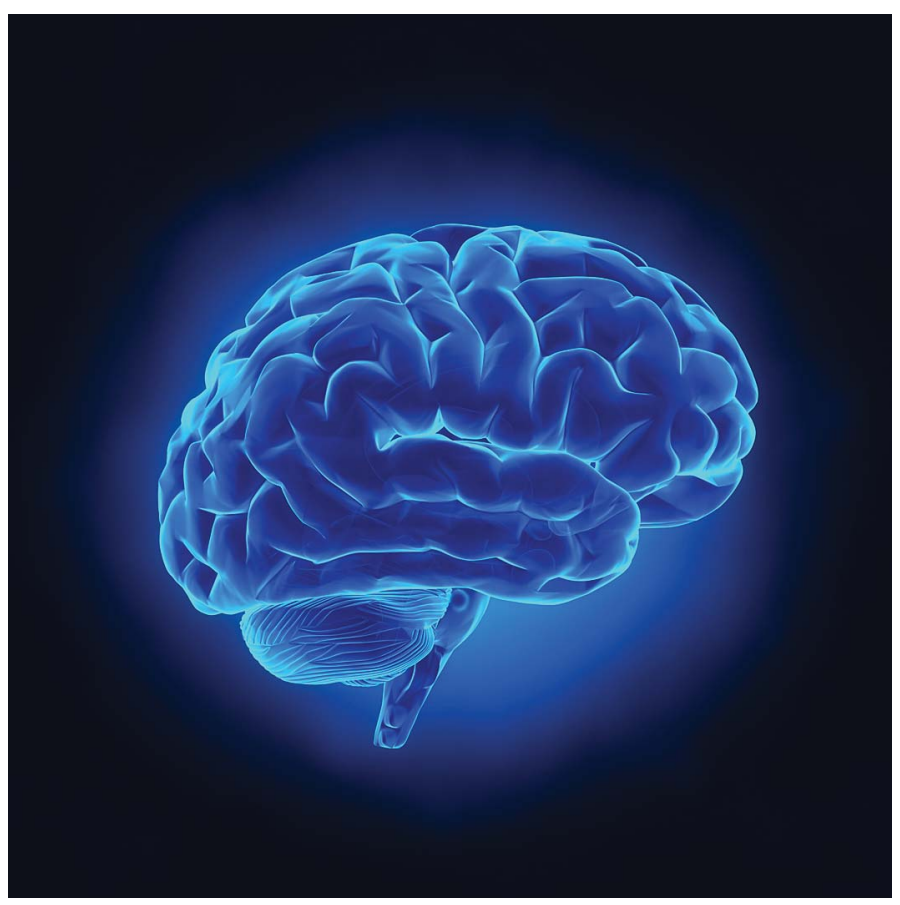
CT for diagnosis of cerebral ischemia in the acute setting. ${ }^{1}$ Diffusion-weighted MRI can show ischemia within an hour of onset of stroke

Fremantle Hospital and Health Service, Fremantle, Western Australia.

Correspondence to: jwatts@globaldial.com 


\section{While MRI remains valuable in the investigation} and management of ischemic stroke, we have identified certain stroke syndromes that are more commonly associated with DWI-negative MRI.

symptoms, and commonly remains positive for up to 14 days following an acute infarct. ${ }^{2,3}$ There is a recognized false-negative rate for DWI changes within this time period in clinically definite strokes, as demonstrated by an increasing number of reports in the literature. ${ }^{4,5}$

All studies in this field report a significantly higher frequency of false-negative diffusionweighted MRI in posterior circulation infarcts. ${ }^{4-7}$ There is only one other large population study assessing which clinically definite stroke syndromes are more likely to be DWI-negative. ${ }^{6}$ Our study aims to identify which clinical stroke syndromes are more frequently associated with false-negative DWI.

\section{METHODS}

The Fremantle Hospital Stroke Unit database was used to retrospectively identify all patients in which the final clinical diagnosis was acute ischemic stroke between November 2005 and June 2011 inclusive. Acute ischemic stroke was diagnosed by experienced stroke neurologists with full access to MRI and other routine investigations. Patients who had MRI as a part of their management were identified. MRI reports of these patients were examined, and patients with no DWI changes were identified for further evaluation. All patients who had MRI more than 14 days from the onset of their symptoms were excluded. Patients with transient ischemic attack (TIA) and patients who were subsequently (either during admission or on follow-up/readmission to hospital) given an alternate diagnosis were also excluded. All patients who had a confirmed diagnosis of acute ischemic stroke with no reported DWI changes had their case notes reviewed by experienced stroke neurologists and their images reviewed by neuroradiologists. If the diagnosis of clinically definite DWI-negative ischemic stroke stood, they were included in the case series.

Prior to MRI, all patients underwent clinical assessment by a stroke neurologist, and had CT brain to exclude hemorrhage. MRI was performed using a $1.5 \mathrm{~T}$ magnetic resonance scanner (Siemens Avanto). Axial DWI/apparent diffusion coefficient (ADC), fluid-attenuated inversion recovery (FLAIR), magnetic resonance angiography (MRA), and T1/T2-weighted sequences were performed in every MRI scan. Acute ischemia was determined based on increased DWI signal and reduced signal on ADC map. Positive DWI was defined by high signal on b1,000 image. DWI had a 5-mm slice thickness with a 2-mm interslice gap.

Time to MRI scan was determined as time from onset of stroke symptoms until MRI scan was performed. Sex, age at presentation, stroke risk factors, NIH Stroke Scale (NIHSS) score at time of scan, stroke syndrome, Oxfordshire classification, evidence of remote stroke on initial scan, and MRI evidence suggestive of small-vessel disease, as assessed by neuroradiologists, were also recorded.

\section{RESULTS}

We identified 996 patients admitted to the Fremantle Hospital Stroke Unit within the designated time period who were diagnosed with acute ischemic stroke. Of these, 701 patients had MRI as part of their management. Thirty-one patients had no DWI changes on MRI. We excluded 15 patients upon further review of case notes, imaging, and follow-up diagnoses (6 patients had functional symptoms, 5 had TIA, and 4 had migraine or other intracranial pathology). This left 16 patients who had clinically definite, DWI-negative acute ischemic stroke. This represented $2.3 \%$ of patients admitted to the Fremantle Stroke Unit with presumed acute ischemic stroke who had MRI. Five were female and 11 male. The median age of this 


\section{Figure A 57-year-old man with lateral medullary syndrome}

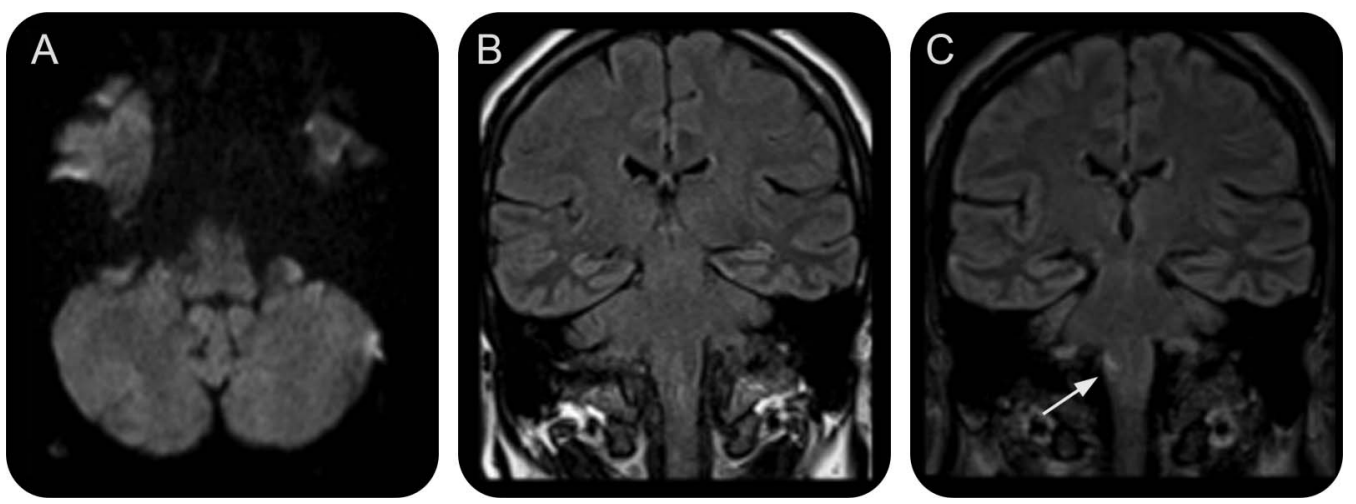

Axial diffusion-weighted imaging on day of symptom onset shows no acute changes (A). Coronal fluid-attenuated inversion recovery (FLAIR) image on the same day (B). Coronal FLAIR image performed 30 days post stroke shows $\mathrm{R}$ medullary lesion (C).

population was 72 years. Median time from symptom onset to MRI was 25 hours, with scans being performed between 4 hours and 194 hours following onset of clinical symptoms. Only 2 patients underwent MRI more than 48 hours after symptom onset. The median NIHSS score of the population was 3. No patients had evidence of remote stroke on MRI. The most common stroke risk factor was hypertension $(\mathrm{n}=11)$. MRI changes in keeping with small-vessel disease were noted in 11 of the 16 cases.

Of the 16 cases, the most common DWI-negative stroke syndromes were ataxic hemiparesis $(\mathrm{n}=5)$ and isolated internuclear ophthalmoplegia (INO) $(\mathrm{n}=5)$. Fifteen of the 16 cases were classified as either posterior circulation or lacunar strokes (table). Follow-up MRI was performed on 4 patients and demonstrated new signal change in 2 of these cases, which was not evident on the initial scan (figure). Neither of the patients with ischemia on repeat MRI had new or progressive neurologic deficits preceding the repeat scans.

\section{DISCUSSION}

While MRI remains valuable in the investigation and management of ischemic stroke, we have identified certain stroke syndromes that are more commonly associated with DWI-negative MRI. This case series identifies 16 cases of DWI-negative stroke, constituting 2.3\% of ischemic stroke patients who had MRI. Almost all cases were classified as either posterior circulation or lacunar stroke, with isolated INO and ataxic hemiparesis being the most common syndromes.

In comparison to other studies, the overall rate of DWI-negative stroke is somewhat low. This is in part likely to reflect the stringent selection of cases in our series, with only clinically definite strokes included and MRI scans initially reported as DWI-negative re-examined by experienced neuroradiologists. Additionally, DWI-positive stroke cases were not reviewed, raising the possibility of false-positive case inclusion. Though clinical stroke phenotype was not examined in DWI-positive patients, other studies have suggested lower rates of lacunar and posterior circulation stroke in Stroke Unit patient cohorts. ${ }^{8-10}$ This suggests significance of the near exclusive representation of posterior circulation and lacunar stroke syndromes in our series.

There are several possible explanations for false-negative DWI stroke syndromes. These hypotheses may be categorized in respect to issues surrounding MRI scanning techniques, size of DWI-negative lesions, and time from symptom onset to scan. In patients with lateral medullary strokes, it is possible that axial DWI did not sufficiently extend inferiorly to identify ischemia. In the case of brainstem infarcts, the local field effect may produce susceptibility artifact, which distorts image analysis and may confound identification of ischemia. In strokes where we would expect small DWI changes, such as isolated INO, the area of ischemia may be too small to produce 


\begin{tabular}{|c|c|c|c|c|c|c|c|c|}
\hline $\begin{array}{l}\text { Oxfordshire } \\
\text { classification }\end{array}$ & Clinical syndrome & $\begin{array}{l}\text { Age, y } \\
\text { (median = 72) }\end{array}$ & Sex & $\begin{array}{l}\text { Stroke } \\
\text { risk factors }\end{array}$ & $\begin{array}{l}\text { Time to scan, } \mathrm{h} \\
\text { (median }=25 \text { hours) }\end{array}$ & $\begin{array}{l}\text { NIHSS at time of } \\
\text { scan (median }=3 \text { ) }\end{array}$ & $\begin{array}{l}\text { Evidence of } \\
\text { remote stroke } \\
\text { on MRI }\end{array}$ & $\begin{array}{l}\text { MRI evidence in } \\
\text { keeping with small- } \\
\text { vessel disease }\end{array}$ \\
\hline POCS & INO & 72 & M & HTN & 194 & 3 & No & Yes \\
\hline POCS & INO & 73 & M & AF, HTN & 29 & 2 & No & Yes \\
\hline POCS & INO & 72 & M & HTN & 18 & 1 & No & Yes \\
\hline POCS & INO & 59 & $\mathrm{~F}$ & HTN & 20 & 1 & No & No \\
\hline POCS & INO & 71 & M & $\mathrm{AF}$ & 25 & 1 & No & No \\
\hline POCS & Lateral medullary & 57 & M & Dyslipidemia & 18 & 4 & No & No \\
\hline POCS & Lateral medullary & 72 & M & T2DM, HTN & 67 & 3 & No & Yes \\
\hline POCS & Lateral medullary & 73 & $\mathrm{~F}$ & HTN & 25 & 3 & No & Yes \\
\hline LACS & Hemiballismus & 83 & M & - & 34 & 3 & No & Yes \\
\hline LACS & Hemiballismus & 65 & M & HTN, dyslipidemia & 4 & 3 & No & Yes \\
\hline LACS & Ataxic hemiparesis & 75 & M & T2DM, HTN & 5 & 4 & No & No \\
\hline LACS & Ataxic hemiparesis & 83 & $\mathrm{~F}$ & HTN & 25 & 3 & No & Yes \\
\hline LACS & Ataxic hemiparesis & 71 & M & HTN & 33 & 2 & No & Yes \\
\hline LACS & Ataxic hemiparesis & 66 & M & - & 12 & 3 & No & Yes \\
\hline LACS & Ataxic hemiparesis & 71 & $F$ & HTN & 26 & 3 & No & No \\
\hline PACS & Dysphasia & 77 & $F$ & IHD & 6 & 5 & No & Yes \\
\hline
\end{tabular}

Abbreviations: $\mathrm{AF}=$ atrial fibrillation; DWI = diffusion-weighted imaging; $\mathrm{HTN}=$ hypertension; $\mathrm{IHD}=$ ischemic heart disease; INO = (isolated) internuclear ophthalmoplegia; $\mathrm{LACS}=$ lacunar stroke; PACS = partial anterior circulation stroke; POCS = posterior circulation stroke; T2DM = type 2 diabetes mellitus. 


\section{Early scanning during a time of critical} ischemia but prior to cytotoxic edema may be a contributory factor to negative DWI in certain

\section{cases.}

adequate DWI signal change. Studies have associated lower NIHSS scores with negative DWI. ${ }^{11}$ This is also suggested in our series. Lower NIHSS scores are more likely to reflect smaller stroke volume. ${ }^{11}$ Additionally, in posterior circulation or lacunar infarcts, ischemia may be missed due to slice thickness or interslice gap. Our MRI stroke protocol uses 5-mm slice thickness with a 2-mm interslice gap for axial DWI scans. This is similar to other studies that quote a range of 1-2 mm interslice gap. ${ }^{4-6}$ Change of positioning of patients may account for cases where repeat MRI was performed and positive signal changes were seen on the repeat scan.

While it is recognized that the rate of DWI-negative strokes increases with greater time to scan, ${ }^{11}$ MRI performed in the acute stage may also have a higher negative rate. ${ }^{1,6}$ Our median time of 25 hours for "time to scan" appears to be a reasonable period to ensure the sensitivity of DWI, reducing the chance that DWI signal is falsely negative due to reperfusion signal reversal or lack of cytotoxic edema. Early scanning during a time of critical ischemia but prior to cytotoxic edema may be a contributory factor to negative DWI in certain cases. The patient with the highest NIHSS score (5) was the only case of anterior circulation stroke. Her clinical presentation suggested a relatively large region of ischemia though DWI performed 6 hours post symptom onset was negative. The patient had repeat MRI performed 7 days poststroke that identified new DWI signal change. It is therefore possible that early reperfusion resulted in normalization of DWI signal.

Technical factors that may aid in reducing false-negative DWI scans include the use of a magnetic resonance scanner with ability to perform perfusion-weighted imaging, identical head positioning for all patients, and decreased interslice gap. The use of 3T diffusion-weighted MRI has potential advantage in diagnosis of subacute (6-24 hours) stroke, ${ }^{12}$ but has not been proven to be advantageous in diagnosis of hyperacute ( $<6$ hours) ischemic stroke lesions. ${ }^{13}$ Ideally, follow-up MRI would have been performed in all patients with initially negative scans, to accurately assess for persistent vs transient negative DWI strokes and to monitor for other pathology.

Our study has identified that certain stroke syndromes, such as ataxic hemiparesis and INO, are more likely to be DWI-negative in the acute setting. Though the clinical assessment of all potential stroke cases is of utmost importance, we suggest extra vigilance for patients with the above presentations. Future prospective studies utilizing more advanced MRI techniques as described above and involving repeat MRI scans may provide further information regarding false-negative DWI in acute ischemic stroke.

\section{REFERENCES}

1. Chalela JA, Kidwell CS, Nentwich LM, et al. Magnetic resonance imaging and computed tomography in emergency assessment of patients with suspected acute stroke: a prospective comparison. Lancet 2007;369:293-298.

2. Schulz UG, Briley D, Meagher T, Molyneux A, Rothwell PM. Diffusion-weighted MRI in 300 patients presenting late with sub-acute transient ischemic attack or minor stroke. Stroke 2004;35: 2459-2465.

3. Schulz UGR, Flossmann E, Francis JM, Redgrave JN, Rothwell PM. Evolution of the diffusion weighted signal and the apparent diffusion coefficient in the late phase after minor stroke. J Neurol 2007;254:375-383.

4. Sylaja PN, Coutts SB, Krol A, Hill MD, Demchuk AM. When to expect negative diffusion-weighted images in stroke and transient ischemic attack. Stroke 2008;39:1898-1900. 
5. Morita S, Masanobu S, lizuka K. False-negative diffusion-weighted MRI in acute cerebella stroke. Auris Nasus Larynx 2011;38:577-582.

6. Oppenheim C, Stanescu R, Dormont D, et al. False-negative diffusion-weighted MR findings in acute ischemic stroke. AJNR Am J Neuroradiol 2000;21:1434-1440.

7. Chen L, Lee W, Chambers BR, Dewey HM. Diagnostic accuracy of acute vestibular syndrome at the bedside in a stroke unit. J Neurol 2011;258:855-861.

8. Ng YS, Stein J, Ning MM, Black-Schaffer RM. Comparison of clinical characteristics and functional outcomes of ischaemic stroke in different vascular territories. Stroke 2007;38:2309-2314.

9. Moulin T, Tatu L, Vuillier F, Berger E, Chavot D, Rumbach L. Role of a stroke data bank in evaluating cerebral infarction subtypes: patterns and outcomes of 1,776 consecutive patients from the Besancon stroke registry. Cerebrovasc Dis 2000;10:261-271.

10. Marti-Vilalta JL, Arboix A. The Barcelona stroke registry. Eur Neurol 1999;41:135-142.

11. Doubal FN, Dennis MS, Wardlaw JM. Characteristics of patients with minor ischemic strokes and negative MRI: a cross-sectional study. J Neurol Neurosurg Psychiatry 2011;82:540-542.

12. Kuhl CK, Textor J, Gieseke J, et al. Acute and subacute ischaemic stroke at high-field-strength (3.0T) diffusion-weighted MR imaging: intraindividual comparative study. Radiology 2005;234:509-516.

13. Rosso C, Drier A, Lacroix D, et al. Diffusion-weighted MRI in acute stroke within the first 6 hours; 1.5 or 3.0 Tesla? Neurology 2010;74:1946-1953.

\section{STUDY FUNDING}

No targeted funding reported.

\section{DISCLOSURES}

The authors report no disclosures relevant to the manuscript. Go to Neurology.org/cp for full disclosures.

\section{Related articles from other AAN physician and patient resources}

\section{Neurology ${ }^{\circledR} \quad \bullet \quad$ www.neurology.org}

A new early and automated MRI-based predictor of motor improvement after stroke July 3, 2012;79:39-46.

\section{Continuum: Lifelong Learning in Neurology ${ }^{\circledR} \quad$ - www.ContinuumJournal.com}

\section{Supranuclear motility}

August 2009;128-149.

Acute ischemic stroke

December 2008.

Neuroimaging ischemia and cerebrovascular disorders

August 2008;19-36.

\section{Neurology Today ${ }^{\circledR} \quad \bullet \quad$ www.neurotodayonline.com}

Medicare Panel: Too little evidence to show amyloid PET scans improve Alzheimer outcomes. March 7, 2013;13:1,36-42. 